\title{
Information Material
}

\section{For readers' attention!}

The publishing house "Naukova dumka" has released monographs of prominent Ukrainian scientists in the fields of computer technologies and mathematical modeling, propagation and diffraction of waves in magnetohydrodynamic and biological systems.

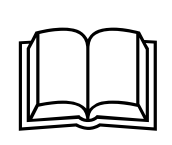

UDK 532.59

Selezov I.T., Kryvonos Yu.G.

Mathematical Methods in Problems of Propagation and Diffraction of Waves

Kiev: Naukova dumka, 2012. $-232 \mathrm{p}$.

Monograph is devoted to urgent problems of the modern theory of wave diffraction. Problems of the theory of hydrodynamic, electromagnetic, magnetoacoustic and elastic wave scattering by local inhomogeneities in unbounded and semi-bounded domains are considered. Analytical and numerical methods of solving problems are presented.

It is recommended for experts in fields of the mechanics of continua, the mathematical physics, applied and calculus mathematics. It will be also useful to teachers, postgraduates and students of corresponding specialities.

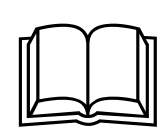

UDK 531/534:57

Selezov I.T., Kryvonos Yu.G.

Wave Problems of Biohydrodynamics and Biophysics

Kiev: Naukova dumka, 2013. - 308 p.

The monograph is devoted to urgent problems of the modern theory of wave biohydrodynamics and biophysics. Problems of the wave propagation in blood vessels, wave phenomena at excitation and propagation of nerve impulses, propagation of medical substances in a tissue and interaction of fermentsubstracts are considered. Analytical and numerical-analytical methods of solving problems are presented.

It is recommended for experts in the field of hemodynamics and biophysics. It will be also useful for teachers, postgraduates and students of corresponding specialities.

ISSN 1064-2315 (C) 2015 by Begell House Inc. 
UDK 532.59

Selezov I.T., Kryvonos Yu.G.

\section{Wave Hyperbolic Models of Disturbance Propagation}

Kiev: Naukova Dumka, 2015. - 172 p.

The monograph is devoted to actual problems of the modern theory of construction of degenerate and generalized hyperbolic wave models that predict the propagation of waves with a finite velocity and their applications. Approaches to construct the singular degeneration in parameters and the generalization of parabolic models are considered. The degeneration of hyperbolic equations on the parameters in the Euclidean space and applications to the propagation of waves in elastic plates and shells are investigated. The parabolic models of ferrohydrodynamics, magnetoelasticity and sedimentation on a bottom surface are generalized. The examples of generalized hyperbolic models of the electromagnetic field and the gas (Maxwell), heat, diffusion, turbulence, probability theory, relativistic hydrodynamics and others are presented. The basic relations of continuum mechanics in curvilinear coordinates are presented.

It is recommended for experts in the field of wave dynamics and physics. It will be also useful for teachers and students in related specialities.

\footnotetext{
About Authors

Selezov Igor Timofeevich is a graduate of Kharkov Aviation University, Doctor of physical and mathematical sciences, Professor, Head of department at the Institute of Hydromechanics of NAS of Ukraine.

E-mail: selezov@yandex.ru

Kryvonos Yuriy Georgievich is a graduate of mechanical and mathematical faculty of Dnepropetrovsk State University, Doctor of physical and mathematical sciences, Professor, Academician of NAS of Ukraine, Deputy Director of V.M.Glushkov Institute of Cybernetics of NAS of Ukraine.
} 